$4 \quad$ PREHOSPITAL RECOGNITION AND ANTIBIOTICS FOR 999 PATIENTS WITH SEPSIS: PROTOCOL FOR A FEASIBILITY STUDY

${ }^{1}$ Chris Moore*, ${ }^{2}$ Jenna Bulger, ${ }^{3}$ Matt Morgan, ${ }^{1}$ Timothy Driscoll, ${ }^{1}$ Alison Porter, ${ }^{1}$ Saiful Islam, ${ }^{4}$ Mike Smyth, ${ }^{4}$ Gavin Perkins, ${ }^{2}$ Bernadette Sewell, ${ }^{3}$ Timothy Rainer, ${ }^{5}$ Prabath Nanayakkara, ${ }^{2}$ Chukwudi Okolie, ${ }^{3}$ Susan Allen, ${ }^{2}$ Greg Fegan, ${ }^{6} J a n$ Davies, ${ }^{7}$ Theresa Foster, ${ }^{3}$ Nick Francis, ${ }^{8}$ Fang Gao Smith, ${ }^{3}$ Gemma Ellis, ${ }^{3}$ Tracy Shanahan, ${ }^{9}$ Robin Howe, 'Samuel Ricketts, ${ }^{2}$ Helen Snooks. 'Welsh Ambulance Services NHS Trust, UK; ${ }^{2}$ Swansea University, UK; ${ }^{3}$ Cardiff and Vale University Health Board, UK; ${ }^{4}$ University of Warwick, UK; ${ }^{5}$ VU University Medical Centre, Netherlands; ${ }^{6}$ Patient Representative, UK; ${ }^{7}$ East of England Ambulance Service NHS Trust, UK; ${ }^{8}$ University of Birmingham, UK; ${ }^{9}$ Public Health UK

\subsection{6/10.1136/bmjopen-2018-EMS.4}

Aim Sepsis is common; it kills at least 44000 people every year in the UK. Early recognition and management of sepsis has been shown to reduce morbidity and mortality of people with sepsis. Paramedics frequently come into contact with patients with sepsis, and are well placed to provide early treatment. This feasibility study aims to find out whether paramedics can collect blood cultures from and administer intravenous (IV) antibiotics to patients with sepsis. We will determine the feasibility, safety and acceptability of our trial design and data collection methods.

Method Paramedics will receive training to assist them to recognise sepsis using a screening tool, obtain blood cultures, and provide IV antibiotics. If sepsis is suspected, paramedics will randomly allocate patients to intervention or usual care using scratchcards. Patients will be followed up at 90 days using linked anonymised data to capture length of hospital admission and mortality. We will also collect self-reported healthrelated quality of life at this time. We will interview ten patients and hold a focus group with paramedics, to find out what they think about the intervention.

Results An intervention development group agreed upon the clinical protocol and training methods. Sixty paramedics have been trained. Patient recruitment will commence 1 st December 2017.

Conclusion At the end of this study we will make a recommendation about whether a fully-powered randomised controlled trial is warranted, and if so, we will develop a proposal for further research funding to answer questions regarding safety and effectiveness for patients, and benefit to the National Health Service.

Conflict of interest None

Funding PhRASe was funded by Health and Care Research Wales, Grant 1191. Chris Moore is the Chief Invesitgator.

\section{PREHOSPITAL ECHOCARDIOGRAPHY DURING CPR IMPACTS TREATMENT DECISIONS IN A DUTCH PHYSICIAN-STAFFED HELICOPTER EMERGENCY MEDICAL SERVICE}

\begin{abstract}
${ }^{1,2} \mathrm{R}$ Ketelaars*, ${ }^{2} \mathrm{C}$ Beekers, ${ }^{1,2} \mathrm{GJ}$ van Geffen, ${ }^{1,2} \mathrm{~N}$ Hoogerwerf. ${ }^{1}$ Radboud university medical centre, Nijmegen, The Netherlands; ${ }^{2}$ HEMS Lifeliner 3, Volkel Air Force Base, Volkel, The Netherlands
\end{abstract}

\subsection{6/10.1136/bmjopen-2018-EMS.5}

Aim Patients in cardiac arrest must receive algorithm-based management such as basic life support and advanced (cardiac) life support (ALS). International guidelines dictate diagnosing and treating any factor that may have caused the arrest or may be complicating the resuscitation. ${ }^{1}$ Ultrasound is recognised to be of potential value in this process and can be used in a prehospital setting. ${ }^{2}$ We aim to evaluate the use of prehospital echocardiography during CPR and its impact on treatment decisions in a Dutch helicopter emergency medical service (HEMS).

Method From February 2014 through November 2016 we conducted an observational study in cardiac arrest patients, of any cause, in whom the Nijmegen HEMS performed CPR with concurrent echocardiography. The physicians adhered to ALS algorithms. Echocardiography was performed simultaneously with interruptions of chest compressions to allow for heart rhythm analysis.

Results In 56 patients 102 ultrasound examinations were documented. Treatment decisions were impacted in 49 patients $(88 \%)$ and in $62(61 \%)$ ultrasound examinations. Overall, we found 78 changes. The changes were termination of CPR in 32 patients (57\%) and continuation hereof in 21 (38\%). Other changes were related to fluid management (14\%), adjustment of drugs and doses (14\%), and choice of receiving hospital $(5 \%)$. Causes of cardiac arrest were trauma $(48 \%)$, cardiac (21\%), medical (14\%), asphyxia (9\%), and other (7\%). Conclusion In conclusion, prehospital ultrasound during CPR in our HEMS significantly impacts patient treatment. Ultrasound images can help explain futile care to caregivers and relatives, even if the sensible decision already is to terminate CPR.

\section{REFERENCES}

1. Soar J, Nolan JP, Bottiger BW, Perkins GD, Lott C, Carli P, et al. European resuscitation council guidelines for resuscitation 2015: Section 3. Adult advanced life support. Resuscitation 2015;95:100-47.

2. Breitkreutz $R$, Price $S$, Steiger $H V$, Seeger $F H$, llper $H$, Ackermann $H$, et al. Focused echocardiographic evaluation in life support and peri-resuscitation of emergency patients: A prospective trial. Resuscitation 2010;81(11):1527-33.

Conflict of interest None

Funding None

\section{DEVELOPMENT AND VALIDATION OF A PRAGMATIC PREHOSPITAL TOOL TO IDENTIFY STROKE MIMIC PATIENTS}

${ }^{1,2} \mathrm{G}$ McClelland ${ }^{*},{ }^{2} \mathrm{H}$ Rodgers, ${ }^{3} \mathrm{D}$ Flynn, ${ }^{2} \mathrm{C}$ Price. ${ }^{1}$ North East Ambulance Service NHS Foundation Trust, UK; ${ }^{2}$ Institute of Neuroscience (Stroke Research Group), Newcastle University, UK; ${ }^{3}$ Institute of Health and Society, Newcastle University, UK

\subsection{6/10.1136/bmjopen-2018-EMS.6}

Aim Stroke mimics (SM) are non-stroke conditions producing stroke-like symptoms. Prehospital stroke identification tools prioritise sensitivity over specificity. ${ }^{1}$ It is estimated that $>25 \%$ of prehospital suspected stroke patients are SM. ${ }^{2}$ Failure to identify SM creates inefficient use of ambulances and specialist stroke services. We developed a pragmatic tool to identify SM amongst suspected prehospital stroke patients. Method The tool was developed using regression analysis of clinical variables documented in ambulance records of suspected stroke patients linked to primary hospital diagnoses (derivation dataset, $\mathrm{n}=1,650,40 \% \mathrm{SM})^{3}$ It was refined using feedback from paramedics $(n=3)$ and hospital clinicians $(n=9)$, and analysis of an expanded prehospital derivation dataset ( $n=3,797,41 \%$ SM (original 1650 patients included)).

Results The STEAM tool combines six variables: 1 point for Systolic blood pressure $<90 \mathrm{mmHg}$; 1 point for Temperature $>38.5^{\circ} \mathrm{C}$ with 
heart rate $>90 \mathrm{bpm}$; 1 point for seizures or 2 points for seizures with known diagnosis of Epilepsy; 1 point for Age $<40$ years or 2 points for age $<30$ years; 1 point for headache with known diagnosis of Migraine; 1 point for FAST-ve. A score of $\geq 2$ on STEAM predicted SM diagnosis in the derivation dataset with $5.5 \%$ sensitivity, 99.6\% specificity and positive predictive value (PPV) of $91.4 \%$. External validation $(\mathrm{n}=1,848,33 \% \mathrm{SM})$ showed $5.5 \%$ sensitivity, 99.4\% specificity and $\mathrm{PPV}$ of $82.5 \%$.

Conclusion STEAM uses common clinical characteristics to identify SM patients with high certainty. The benefits of using STEAM to reduce SM admissions to stroke services need to be weighed up against delayed admissions for stroke patients wrongly identified as SM.

\section{REFERENCES}

1. Rudd $\mathrm{M}$, Buck D, Ford GA, Price Cl. A systematic review of stroke recognition instruments in hospital and prehospital settings. Emerg Med J 2016;33:818-822.

2. McClelland $G$, Rodgers $H$, Flynn D, Price C. The frequency and diagnosis of stroke mimics in prehospital and hospital studies: A systematic literature review (poster abstract). International Journal of Stroke 2016;11(4S):30-31.

3. McClelland G, Rodgers H, Flynn D, Price C. Development of a pre-hospital assessment to identify stroke mimic conditions (poster abstract). Emerg Med J 2017;34 (10):PP14.

Conflict of interest None declared

Funding The Stroke Association Postgraduate Fellowship (TSA PGF 2015-01) North East Ambulance Service Trust Fund.

\section{WE NEED SUPPORT!}

${ }^{1,2} \mathrm{~A}$ Hörberg* ${ }^{*}{ }^{3} \mathrm{M}$ Jirwe, ${ }^{1,4} \mathrm{~S}$ Kalén, ${ }^{1,2,5} \mathrm{~V}$ Vicente, ${ }^{2,3} \mathrm{~V}$ Lindström. ${ }^{1}$ Karolinska Institute, Dep of Clinical Science and Education, Södersjukhuset; ${ }^{2}$ Academic EMS Stockholm, Sweden; ${ }^{3}$ Karolinska Institute, Dep of Neurobiology Care Sciences and Society, Division of Nursing; ${ }^{4}$ Stockholm City Council; ${ }^{5}$ The ambulance medical service in Stockholm (AISAB), Sweden

\subsection{6/10.1136/bmjopen-2018-EMS.7}

Aim New and inexperienced emergency medical service (EMS) professionals lack important experience. To prevent medical errors and improve retention there is an urgent need to identify ways to support new professionals during their first year in the EMS.

Method A classical Delphi technique in four rounds was used. A purposeful sample and snowball technique was used and generated an expert panel of 32 registered nurses with 12-36 months of EMS experience. In round one telephone interviews were undertaken to identify what support professionals new to the EMS desire during their first year. Content analysis of the transcribed interviews yielded 70 items which were developed into a questionnaire. In subsequent three rounds the experts graded each item in terms of perceived importance on a 5-graded likert scale. Consensus level was set at 75 percent.

Results Desirable support was categorised into eight areas: Support from practical skills exercises, support from theoretical knowledge, support from experiences based knowledge, theoretical support, support from an introduction period, support from colleagues and work environment, support from management and organisation and other support. The experts agree on the level of importance on 64 items regarding desirable support. Of these, 63 items were considered important, graded 4 or 5 . One item was considered not important, graded 1 or 2 .

Conclusion Even with requested formal competence of the professionals, the EMS context poses challenges where a wide variety of desirable forms of support is needed. Support structures should address both personal and professional levels and be EMS context oriented.

Conflict of interest None

Funding Academic EMS in Stockholm.

\section{RAPID ANALGESIA FOR PREHOSPITAL HIP DISRUPTION (RAPID)}

${ }^{1}$ Jenna Bulger*, ${ }^{2}$ Alan Brown, ${ }^{1}$ Bridie A Evans, ${ }^{1}$ Greg Fegan, ${ }^{3}$ Simon Ford, ${ }^{3}$ Katy Guy, ${ }^{2}$ Sian Jones, ${ }^{4}$ Leigh Keen, ${ }^{1}$ Ashrafunnesa Khanom, ${ }^{3}$ lan Pallister, ${ }^{4}$ Nigel Rees, ${ }^{1}$ lan T Russell, ${ }^{1}$ Anne C Seagrove, 'Helen A Snooks. 'Swansea University Medical School, UK; ${ }^{2}$ Lay representatives, UK; ${ }^{3}$ Abertawe Bro Morgannwg University Health Board, UK; ${ }^{4}$ Welsh Ambulance Services NHS Trust, UK

\subsection{6/10.1136/bmjopen-2018-EMS.8}

Aim Pain relief in prehospital care is a challenge in trauma, especially for those with hip fractures, whose injuries are difficult to immobilise and whose long term outcomes may be adversely affected by administration of opiates. Fascia Iliaca Compartment Block (FICB) is routinely undertaken by hospital clinicians, but has not been fully evaluated for use by paramedics in prehospital care.

Method Nineteen paramedics were trained; they randomly allocated eligible patients to trial arms using audited scratchcards. Patients were followed up to assess availability of outcomes including quality of life, length of admission, pain scores, and adverse events. Findings were analysed against prespecified progression criteria.

Results Seventy-one patients were randomised by paramedics (28/6/16 - 31/7/17); 57 consented to follow up. The only outcome which reached a statistically significant difference between groups was the proportion of participants who received morphine (38\% difference between groups 95\% CI: -61.88 to -15.79 ). There was a difference of approximately nine days in the length of admission between trial arms (mean difference 9.12 (95\% CI: -20.51 to 2.27 ).

Conclusion RAPID met its pre-specified progression criteria; a funding application for a fully-powered RCT will therefore be submitted. We will consider the use of length of stay as the primary outcome, as findings indicated a difference between groups without reaching statistical significance.

Conflict of interest None

Funding Health and Care Research Wales, Research for Patient and Public Benefit.

\section{THE SAME BUT DIFFERENT - VARIATIONS IN STAKEHOLDERS' PRIORITIES, VIEWS ON RANDOMISATION AND FUNDING OF PREHOSPITAL CRITICAL CARE FOR OUT-OF-HOSPITAL CARDIAC ARREST}

\footnotetext{
${ }^{1,2}$ Johannes von Vopelius-Feldt*, ${ }^{2}$ Janet Brandling, ${ }^{1,2}$ Jonathan Benger. ${ }^{1}$ Academic Department of Emergency Care, University Hospitals Bristol; ${ }^{2}$ Emergency Medicine and Critical Care Research Group, University of the West of UK
}

\subsection{6/10.1136/bmjopen-2018-EMS.9}

Aim Prehospital critical care for out-of-hospital cardiac arrest (OHCA) is a complex and largely unproven intervention. During research to examine this intervention, we noted significant differences in stakeholders' views about research, 